ACTA MYCOLOGICA

Vol. 44 (2): 265-273

2009
Dedicated to Professor Krystyna Czyżewska

in honour of 40 years of her scientific activity

\title{
Some new records of Verrucaria from Beskid Niski Mts.
}

\author{
BEATA KRZEWICKA \\ Laboratory of Lichenology, W. Szafer Institute of Botany \\ Polish Academy of Sciences, Lubicz 46 \\ PL-31-512 Kraków, b.krzewicka@botany.pl
}

Krzewicka B.: Some new records of Verrucaria from Beskid Niski Mts. Acta Mycol. 44 (2): 265-273, 2009.

Based on the historical collections of J. Motyka and J. Nowak deposited in LBL and KRAM herbaria 26 species of Verrucaria s.l. are reported from Beskid Niski Mts. Verrucaria elaeina is reported from Poland for the first time, whereas as much as 23 species are new to the region.

Key words: new records, freshwater species, Verrucaria elaeina, Hydropunctaria, Parabagliettoa, Placopyrenium, S Poland

\section{INTRODUCTION}

The Beskid Niski Mts. are situated in the eastern part of the Western Carpathians, in the greatest depression of the Carpathian arc, and they bridge the Western Carpathians with the Eastern Carpathians. The first data about lichens in this area were reported from Kornuty nature reserve by Sulma (1936). He listed ca 30 species, mainly macrolichens. Whereas, the first note on Verrucaria in this mountain range was published by Olech (1974). In the western part of Beskid Niski Mts. she recorded 226 species of lichens, including one species of Verrucaria, V. nigrescens. At that time an intensive field study in this area were carried out also by J. Kiszka, R. Kozik (KRAP) and J. Nowak (KRAM), and continued by M. Olech (KRA). Among these herbarium collections only the material gathered by M. Olech was partly published so far (Olech 1974). However, the largest collection especially of pyrenocarpous lichens from Beskid Niski Mts., including Verrucaria is available at the KRAM herbarium.

Additionally, during the author's studies on Verrucaria genus in Poland an interesting historical material from Kąclowa in Beskid Niski Mts. was found in LBL herbarium. It was collected in the first part of $20^{\text {th }}$ century by J. Motyka. Both the oldest 
(LBL) and the largest (KRAM) collection of Verrucaria from Beskid Niski Mts. was examined during the course of this study.

\section{MATERIAL AND METHODS}

The study is based on the lichen material collected by Janusz Nowak in eastern part of the Beskid Niski Mts. in the years 1974-1975 and 1979 (KRAM, Kraków) and on material collected by Józef Motyka in Kąclowa near Grybów in 1924, 1926, 1954 and 1965 (LBL, Lublin).

The localities are coded using ATPOL grid square system (changed by Cieśliński \& Fałtynowicz, eds 1993 and are presented on a map (Fig. 1). The names of localities follows the tourist map "Beskid Niski i Pogórze" scale 1:125 00. Observation and measurements of spores and ascomata structures were made on sections mounted in water. Nomenclature of species is based on taxonomic studies by Orange (2000, 2004), Thüs (2002), Navarro-Rosinés et al. (2007), Gueiden et al. (2009), and Krzewicka (2009).

\section{RESULTS AND DISCUSSION}

Based on the lichen collections 26 species of Verrucaria s.l. were determined. Two species belong to newly described genera: Hydropunctaria, H. rheitrophila, and Parabagliettoa, P. cyanea (Gueiden et al. 2009). Another one is currently a member of Placopyrenium, $P$. fuscellum (Navarro-Rosinés et al. 2007). Twenty three represent the genus Verrucaria, V. aethiobola, V. andesiatica, V. aquatilis, V. dolosa, V. elaeina, V. elaeomelaena, V. funckii, V. hydrela, V. latebrosa, V. margacea, V. minuta, V. muralis, V. murina, V. nigrescens, V. ochrostoma, V.obfuscans, V. polysticta, V. praetermissa, V. rupestris, V. sublobulata, submersella, V. umbrinula, V. viridula (see also Appendix).

The species Verrucaria elaeina is for the first time reported from Poland, whereas all the other records except of V. nigrescens, V. polysticta and P. fuscellum (Olech 1974; Krzewicka 2009) are new to the Beskid Niski Mts.

The species of Verrucaria were collected in the study area in dry and sunny places on rocks or pebbles in meadows and grazing lands, and on pebbles in watercoursebeds, from splash to submerged zones. These epilithic lichens grow on calcareous or non-calcareous substrata, e.g., on concrete, sandstone with or without calcium carbonate or on bricks. They are often associated with Aspicilia, Caloplaca, Candelariella, Lecanora and Thelidium spp. According to Nascimbene et al. (2007) lichens belong to three main ecological guilds on the basis of their water requirements, 1) species of perennially inundated habitats; 2 ) semi-aquatic species of periodically inundated habitats, and 3) terrestrial species. All guilds are represented among Verrucaria s.l. species occurring in the study area. 

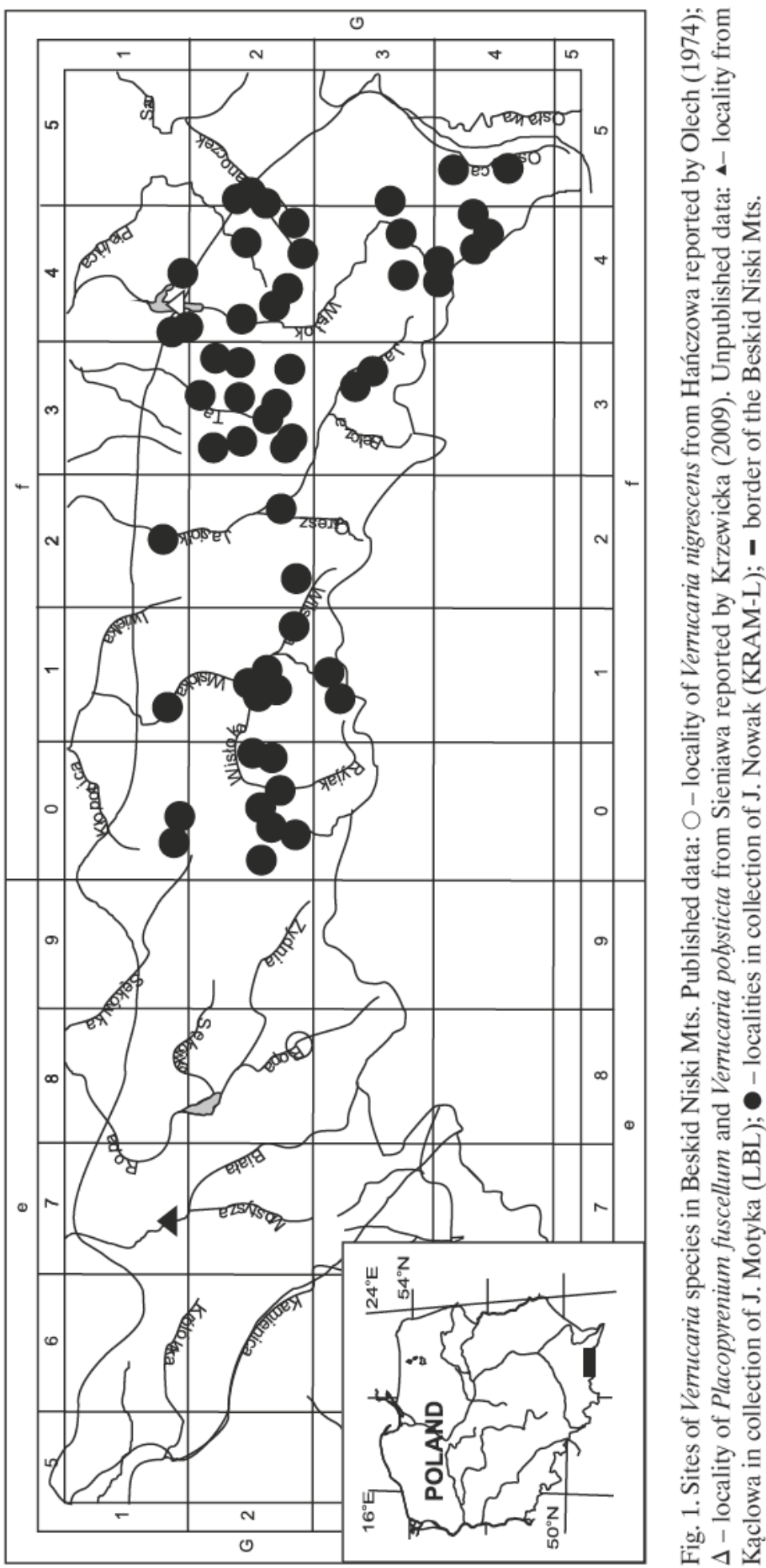
In total eleven freshwater Verrucaria occurring in or by watercourse were distinguished in the area: Verrucaria aethiobola, $V$. andesiatica, V. aquatilis, V. funckii, $V$. hydrela, V. latebrosa, V. margacea, V. praetermissa, V. sublobulata, V. submersella and Hydropunctaria rheitrophila, along with some other associated lichen species, for example Bacidia inundata, Thellidium aquatiqum, Th. minutulum, Th. zwackhii. Verrucaria aquatilis, V. funckii, V. hydrela, and $H$. rheitrophila which are characteristic to the submerged zone and $V$. elaeomelaena, and $V$. latebrosa characteristic to the splash zone represent species of perennially inundated habitats. The most frequent species of this guild is $V$. aquatilis which was collected nearly in all examined streams of Beskid Niski Mts. To the semi-aquatic species of periodically inundated habitats belong V. aethiobola, V. andesiatica, V. margacea, V. praetermissa, V. sublobulata, and $V$ submersella. The most frequent species of this guild is $V$. praetermissa which was found in all examined streams in the area. It is worth mentioning this particular species occurs also on permanently inundated substrata. Most of the freshwater species are in fact amphibious organisms, and most of them being submerged during only a part of year mainly in spring or autumn when the water level is higher.

Terrestrial lichens were represented by 15 species of Verrucaria s.l., such as Parabagliettoa cyanea, V. dolosa, V. elaeina, V. minuta, V. muralis, V. murina, V.nigrescens, V. ochrostoma, V. obfuscans, V. procopii, V. rupestris, V. umbrinula, V. viridula, and including two parasitic species $V$. polysticta and Placopyrenium fuscellum that have been previously reported from Beskid Niski Mts. (Krzewicka 2009). These predominantly epilithic lichens occurred in the study area mainly on pebbles in meadows and grazing lands, whereas on anthropogenic substrata such as on bridges, walls and rock fences they were less frequent.

\section{THE NEW RECORD FROM POLAND}

Verrucaria elaeina Borrer, in: W. J. Hooker, English Botany Supplement 1: text to plate 2623, fig. 2 (1830).

This species is characterized by the typically pale, epilithic, usually cracked, nongelatinous thallus, the more or less conical involucreum, and the medium-size of ascospores, 16.5-19.4-22.5 × 7.0-8.0-9.0 $\mu \mathrm{m}$. It resembles $V$ praetermiss $\mathrm{in}$ the epilithic, non-gelatinous, generally weakly pigmented thallus, somewhat conical involucreum, tolerance of shade, and in the size and shape of the ascospores. V. elaeina differs however, due to the more prominent perithecia, lack of a black basal layer (which is usually well-developed in V praetermissa), slightly smaller ascospores, and lack of a perispore.

This species has been misunderstood in Europe for last 150 years (Orange 2000), including Poland where it was mistakenly determined as $V$. guestphalica auct.

According to Orange (2000) the species grows on a variety of substrata, including limestone, calcareous mudstone, sandstone, mortar, concrete and bricks; in natural and semi-natural habitats including cliffs, woodland, and occasionally beside streams, also on paths, walls and debris in gardens, by disused railways and on ruined buildings; often on stone embedded in the ground, and usually in shade. It typically 
occurs in species-poor communities; it often grows in abundance, but with only a few associated lichen.

In Beskid Niski Mts. the species seems to be frequent and occupies sandstone pebbles and banks of streams, mainly in the upper part of watercourse banks on sheltered and humid rocks (see Appendix). In Europe it is known from Great Britain, Ireland, Norway, Germany, Switzerland, and Austria (Orange 2000; Santesson et al. 2004).

Acknowledgements. The author is grateful to Lucyna Śliwa the Curator of KRAM-L herbarium, and to Wiesław Mułenko the Curator of LBL herbarium for kindly allowing me to study Verrucaria material, as well as to the anonymous reviewer for suggestions on the manuscript. Thanks is also due to Anetta Wieczorek, and Paweł Czarnota for their help with the draft of the map.

\section{REFERENCES}

Cieśliński S., Fałtynowicz W. (eds). 1993. Atlas of the geographical distribution of lichens in Poland. 1. W. Szafer Institute of Botany, Polish Academy of Sciences, Kraków.

Gueidan C., Savić S., Thüs H., Roux C., Keller C., Tibell L., Prieto M., Heiðmarsson S., Breuss O., Orange A., Fröberg L., Amtoft Wynns A., Navarro-Rosinés P., Krzewicka B., Pykälä J., Martin G., Lutzoni F. 2009. Generic classification of the Verrucariaceae (Ascomycota) based on molecular and morphological evidence: recent progress and remaining challenges. Taxon 58 (1): 184-208.

Krzewicka B. 2009. The 'Verrucaria fuscella group' in Poland with some nomenclatorial remarks. Acta Soc. Bot. Pol. 78 (3): 229-234.

Nascimbene J., Thüs H., Marini L., Nimis P.L. 2007. Freshwater lichens in springs of the eastern Italian Alps: floristics, ecology and potential for bioindication. Ann. Limol. Int. J. Lim. 43 (4): 285-292.

Navarro-Rosinés P., Roux C., Gueidan C. 2007. La genroj Verrucula kaj Verruculopsis (Verrucariaceae, Verrucariales). Bull. Soc. Linn. Provence 58: 133-180.

Olech M. 1974. Materiały do flory porostów Beskidu Niskiego. Zesz. Nauk. Uniw. Jagiellon., Prace Bot. 2: $181-200$.

Orange A. 2000. Verrucaria elaeina, a misunderstood European lichen. Lichenologist 32 (5): 411-422.

Orange A. 2004. The Verrucaria fuscella group in Great Britain and Ireland. Lichenologist 36: 173-182.

Santesson R., Moberg R., Nordin A., Tønsberg T., Vitikainen O. 2004. Lichen-forming and Lichenicolous Fungi of Fennoscandia. Museum of Evolution, Uppsala University, Uppsala.

Sulma T. 1936. Kornuty - rezerwat na Łemkowszczyźnie. Wyspowe stanowisko kosodrzewiny (Pinus mughus Scop.) w Beskidach Niskich. Ochr. Przyr. 16: 57-73.

Thüs H. 2002. Taxonomie, Verbreitung und Ökologie silicoler Süßwasserflechten im außeralpinen Mitteleuropa. Biblioth. Lichenol. 83: 1-214.

\section{APPENDIX - LIST OF SPECIES}

Hydropunctaria rheitrophila (Zschacke) Keller, Gueidan \& Thüs [=Verrucaria rheitrophila] - ATPOL grid square Gf 20 - Rozstajne, stream between Cyrla Mt. and 601 peak, 480 m, 21 Sept. 1979, J. Nowak (KRAM-L 32617); Krempna, Las Słodki forest, stream on N slope of Cyrla, 500 m, 8 Oct. 1979, J. Nowak (KRAML 32620) together with $V$. praetermissa. Gf 23 - stream between Dział Mt. near Szklary and Obłaz Mt. near Daliowa, 520 m, 11 Sept. 1974, J. Nowak (KRAM-L 33419b). Gf 44 - Wisłok Wielki Górny, stream Jamiska on W slope of Pasika Mt., 675 m, 22 June 1974, J. Nowak (KRAM-L 33233) together with V. sublobulata (KRAM-L 33232), and V. margacea; Wisłok Górny, Wisłok river, near Ka- 
nasiówka Mt., 625 m, 22 June 1974, J. Nowak (KRAM-L 33405) together with V. aquatilis.

Parabagliettoa cyanea (A. Massal.) Gueidan \& Cl. Roux [=Verrucaria cyanea - Gf 23 - valley of Bołucianka stream near Rymanów, 430 m, 15 Sept. 1974, J. Nowak (KRAM-L 33425).

Placopyrenium fuscellum (Turner) Gueidan \& Cl. Roux [=Verrucaria fuscella $]$ - Gf 14 - Sieniawa, by Wisłok river on sandstone, 315 m, 20 Aug. 1974, J. Nowak (KRAM-L 33306). Gf 45 - Komańcza Letnisko, 460 m, 18 June 1974, J. Nowak (KRAM-L 33259).

Verrucaria aethiobola Wahlenb. - Ge 17 - Kąclowa, 26 July 1926, J. Motyka (LBL). Gf 34 - Wisłok Górny, stream on W slope of Jasienina peak, by Izwor stream, 550 m, 23 June 1974, J. Nowak (KRAM-L 33248); Wisłok Górny, Wisłok stream below Bieszczad hill, 550 m, 22 June 1974, J. Nowak (KRAM-L 33312).

Verrucaria andesiatica Servít - Ge 17 - Kąclowa, 26 July 1926, J. Motyka (LBL).

Verrucaria aquatilis Mudd - Gf 10 - Świerzowa Ruska near Świątkowa, 460 m, 7 Sept. 1979, J. Nowak (KRAM-L 33356) together with V. praetermissa. Gf 20 Ryjak river by Rozstajne, 440 m, 21 Sept. 1979, J. Nowak (KRAM-L 33378); Nieznajowa by ruins of the church, Zawoja river, 460 m, 9 Oct. 1979, J. Nowak (KRAM-L 32556, 32556, 32251, 32253); Czarne by Nieznajowa, in stream, 470 m, 4 Oct. 1979, J. Nowak (KRAM-L 32452, 32449, 32448, 32449, 32452). Gf 21 Ostryszne village by Krempna, on bank of Wisłoka river, 350 m, 19 Sept. 1979, J. Nowak (KRAM-L 33466) together with V. praetermissa; Olchowiec near Polany in stream, 450 m, 5 Oct. 1979, J. Nowak (KRAM-L 32501, 32447, 33020); Krempna, stream by Huta Krempska 390 m, 18 Sept. 1979, J. Nowak (KRAM-L 32223); Polany by Krempna, 350 m, 19 Sept. 1979, J. Nowak (KRAM-L 32770); Huta Krempska by Krempna, 420 m, 18 Sept. 1979, J. Nowak (KRAM-L 32772) together with $V$. praetermissa. Gf 23 - Wisłok river near Tarnawka, 440 m, 13 Sept. 1974, J. Nowak (KRAM-L 33429); stream between Dział Mt. by Szklary and Obłaz Mt. near Daliowa, 520 m, 11 Sept. 1974, J. Nowak (KRAM-L 33418, 33420); Wisłoczek, N slope of Jawornik Mt., 620 m, 21 Aug. 1974, J. Nowak (KRAM-L 33264). Gf 24 - in valley of Pielnica stream, Roztoki near Wola Sękowa, 445 m, 9 Oct. 1974, J. Nowak (KRAM-L 33304); Pasmo Bukowicy Mts., Puławy near Wisłok river, 12 Oct. 1974, J. Nowak (KRAM-L 33298). Gf 33 - Wola Wyżna near Jaśliska, Jasiołka stream, 510 m, 14 Sept. 1974, J. Nowak (KRAM-L 33414) together with V. praetermissa. Gf 44 - Wisłok Górny, Wisłok river, near Kanasiówka Mt., 625 m, 22 June 1974, J. Nowak (KRAM-L 33323, 33405); Dołżyca near Komańcza, stream between Średni Garb Mt. and Danawa Mt., 650 m, 20 June 1974, J. Nowak (KRAM-L 33388).

Verrucaria dolosa Hepp - Ge 17 - Kąclowa, 500 m, 20 July 1920 and 16 July 1926, J. Motyka (LBL). Gf 20 - Czarne near Nieznajowa, 470 m, 4 Oct. 1979, J. Nowak (KRAM-L 32505, 33017). Gf 21 - Ostryszne near Krempna, 340 m, 19 Sept. 1979, J. Nowak (KRAM-L 33477). Krempna, 380 m, 18 Sept. 1979, J. Nowak (KRAM-L 32558).

Verrucaria elaeina Borrer - Ge 17 - Kąclowa, 1954, J. Motyka (LBL). Gf 10 Majdan between Bartne and Świerzowa Ruska, 540 m, 7 Sept. 1979, J. Nowak (KRAM-L 32528). Gf 14 - Odrzechowa, Czernisławka river, 23 Oct. 1974, J. Nowak (KRAM-L 33427); near Mymoń by Rymanów, 305 m, 23 Aug. 1974, J. 
Nowak (KRAM-L 33279, 33308). Gf 20 - Żydowskie near Krempna, 570 m, 6 Oct. 1979, J. Nowak (KRAM-L 32586). Kotań near Krempna, 470 m, 17, Sept. 1979, J. Nowak (KRAM-L 32618); Czarne near Nieznajowa, 470 m, 4 Oct. 1979, J. Nowak (KRAM-L 32502). Gf 21 - Olchowiec near Polany in stream, 450 m, 5 Oct. 1979, J. Nowak (KRAM-L 33021); stream in Wielki Las forest near Krempna, 390 m, 18 Sept. 1979, J. Nowak (KRAM-L 32880). Gf 23 - Rudawa stream near Królik Polski, 500 m, 21 Aug. 1974, J. Nowak (KRAM-L 33438). Gf 24 - Puławy by Wisłok river, valley of stream between Kiczera Mt. and Kiczerka Mt., 450 m, 10 Oct. 1974, J. Nowak (KRAM-L 33049, 33293). Gf 25 - Pasmo Bukowicy Mts., Nagórzany near Bukowsko, Sanoczek stream, 425 m, 24 Oct. 1974, J. Nowak (KRAM-L 33463) together with V. praetermissa. Gf 44 - Dołżyca near Komańcza, stream between Średni Garb Mt. and Danawa Mt., 650 m, 20 June 1974, J. Nowak (KRAM-L 33247) together with V. elaeomelaena. Gf 45 Dołżyca near Komańcza village by Średni Garb Mt., 540 m, 20 June 1974, J. Nowak (KRAM-L 33398); Osławica, on bank Osławica river, 520 m, 21 June 1974, J. Nowak (KRAM-L 33322).

Verrucaria elaeomelaena (A. Massal.) Arnold - Gf 21 - Huta Krempska by Krempna, 420 m, 18 Sept. 1979, J. Nowak (KRAM-L 32772) together with V. praetermissa. Gf 44 - Dołżyca near Komańcza, stream between Średni Garb Mt. and Danawa Mt., 650 m, 20 June 1974, J. Nowak (KRAM-L 33247).

Verrucaria funckii (Spreng.) Zahlbr. - Gf 44 - Wisłok Górny, Wisłok river, near Kanasiówka Mt., 625 m, 22 June 1974, J. Nowak (KRAM-L 33323) together with V. aquatilis.

Verrucaria hydrela Ach. - Gf 20 - Nieznajowa by the ruins of church, $460 \mathrm{~m}, 9$ Oct. 1979, J. Nowak (KRAM-L 32253) together with V. aquatilis. Gf 21 - Olchowiec near Polany in stream, 450 m, 5 Oct. 1979, J. Nowak (KRAM-L 33020) together with V. aquatilis. Gf 44 - Wisłok Górny, Wisłok river, near Kanasiówka Mt., 625 m, 22 June 1974, J. Nowak (KRAM-L 33405) together with V. aquatilis; Wisłok Wielki Górny, Jamiska stream W-slope of Pasika Mt., 675 m, 22 June 1974, J. Nowak (KRAM-L 33232) together with V. margacea; Dołżyca near Komańcza, stream between Średni Garb Mt. and Danawa Mt., 650 m, 20 June 1974, J. Nowak (KRAM-L 33237) together with V. pretermissa.

Verrucaria latebrosa Körb. - Ge 17 - Kąclowa, 1954, J. Motyka (LBL). Gf 12 - bank of Jasionka stream near Dukla, 340 m, 10 Sept. 1974, J. Nowak (KRAM-L 33422). Gf 21 - Krempna, stream by Huta Krempska, 390 m, 18 Sept. 1979, J. Nowak (KRAM-L 32230); Huta Krempska by Krempna, 420 m, 18 Sept. 1979, J. Nowak (KRAM-L 33498, 32828, 33498). Gf 22 - Wilsznia stream near Tylawa, 490 m, 5 Oct. 1975, J. Nowak (KRAM-L 32480, 32481) together with V. praetermissa. Gf 23 - valley of Wisłoczek river near, 490 m, 21 Aug. 1974, J. Nowak (KRAML 32274). Gf 44 - Dołżyca near Komańcza, stream between Średni Garb Mt. and Danawa Mt., 650 m, 20 June 1974, J. Nowak (KRAM-L 33400). Gf 45 Osławica, on bank Osławica river, 520 m, 21 June 1974, J. Nowak (KRAM-L 33236); Dołżyca near Komańcza, stream on slope of Średni Garb Mt., 540 m, 20 June 1974, J. Nowak (KRAM-L 33400).

Verrucaria margacea (Wahlenb.) Wahlenb. - Gf 20 - Nieznajowa near Świątkowa, Las Spełzły forest, 640 m, 4 Oct. 1979, J. Nowak (KRAM-L 33016). Gf 21 Olchowiec near Polany, in stream, 450 m, 5 Oct. 1979, J. Nowak (KRAM-L 32447) 
together with $V$. praetermissa. Gf 44 - Wisłok Wielki Górny, Jamiska stream, W slope of Pasika Mt., 675 m, 22 June 1974, J. Nowak (KRAM-L 33319, 33232).

Verrucaria minuta (Hepp) Zschacke - Gf 11 - Kąty near Nowy Żmigród, 330 m, 25 Oct. 1979, J. Nowak (KRAM-L 33489).

Verrucaria muralis Ach. - Ge 17 - Kąclowa, 19 and 21 July 1926, and 1954, J. Motyka (LBL). Gf 20 - Czarne near Nieznajowa, 540 m, 4 Oct. 1979, J. Nowak (KRAM-L 32479, 33017); Rozstajne near Krempna, 430 m, 6 Sept. 1979, J. Nowak (KRAM-L 33365). Gf 22 - Wilsznia near Tylawa, 540 m, 5 Oct. 1979, J. Nowak (KRAM-L 32451). Gf 23 - valley of Bołucianka stream near Rymanów, 430 m, 15 Sept. 1974, J. Nowak (KRAM-L 33424). Gf 24 - W slope of Tokarnia peak, 720 m, 19 June 1974, J. Nowak (KRAM-L 33252).

Verrucaria murina Leight. - Gf 21 - Krempna, near Huta Krempska, 380 m, 18 Sept. 1979, J. Nowak (KRAM-L 32524). Gf 31 - Ciechania near Krempna, 590 m, 10 Oct. 1979, J. Nowak (KRAM-L 32882).

Verrucaria nigrescens Pers. - Ge 17 - Kąclowa, 20 July 1924 and 10 July 1926, J. Motyka (LBL). Gf 14 - Sieniawa, by Wisłok river on sandstone, 20 Aug. 1974, J. Nowak (KRAM-L 33329). Gf 23 - valley of Bołucianka stream near Rymanów, 430 m, 15 Sept. 1974, J. Nowak (KRAM-L 33425). Gf 31 - Ciechania near Krempna, 500 m, 10 Oct. 1979, J. Nowak (KRAM-L 32769).

Verrucaria ochrostoma (Borrer ex Leight.) Trevis. - Gf 11 - Kąty near Nowy Żmigród, 400 m, 19 Sept. 1979, J. Nowak (KRAM-L 32751). Gf 23 - Wisłoczek near Rymanów Zdrój, 540 m, 21 Aug. 1974, J. Nowak (KRAM-L 33436).

Verrucaria obfuscans Nyl. - Gf 11 - Kąty near Krempna, 310 m, 11 June 1980, J. Nowak (KRAM-L 32645). Gf 22 - on the bank of Jasiołka river near Dukla, 340 m, 10 Sept. 1974, J. Nowak (KRAM-L 33423).

Verrucaria polysticta Borrer - Gf 14 - Sieniawa, by Wisłok river on sandstone, 315 m, 20 Aug. 1974, J. Nowak (KRAM-L 33329).

Verrucaria praetermissa (Trevisan) Anzi - Ge 17 - Kąclowa, 22 July 1926, J. Motyka (LBL). Gf 10 - Świerzowa Ruska near Świątkowa, 460 m, 7 Sept. 1979, J. Nowak (KRAM-L 33356, 33220). Gf 20 - Ryjak river by Rozstajne, 440 m, 21 Sept. 1979, J. Nowak (KRAM-L 33376); Rozstajne, stream between Cyrla Mt. and 601 peak, 480 m, 21 Sept. 1979, J. Nowak (KRAM-L 32617); Krempna, Las Słodki forest, stream on N slope of Cyrla, 500 m, 8 Oct. 1979, J. Nowak (KRAM-L 32620); Czarne by Nieznajowa, in stream, 470 m, 4 Oct. 1979, J. Nowak (KRAML 32448). Gf 21 - Ostryszne villege by Krempna, on bank of Wisłoka river, 350 m, 19 Sept. 1979, J. Nowak (KRAM-L 33466); Olchowiec near Polany in stream, 450 m, 5 Oct. 1979, J. Nowak (KRAM-L 32500, 32447, 32447, 33018); Krempna, Krempna stream, 390 m, 18 Sept. 1979, J. Nowak (KRAM-L 32231, 32225); Huta Krempska, 420 m, 18 Sept. 1979, J. Nowak (KRAM-L 32772). Gf 22 - Wilsznia stream near Tylawa, 490 m, 5 Oct. 1975, J. Nowak (KRAM-L 32481). Gf 23 stream between Dział Mt. by Szklary and Obłaz Mt. near Daliowa, 520 m, 11 Sept. 1974, J. Nowak (KRAM-L 33419, 33416, 33417); Dolina Wołtuszowej valley near Rymanowa Zdrój town, stream near Kopiec Mt., 470 m, ? Sept. 1974, J. Nowak (KRAM-L 33305); stream between Putyska Mt. and Dział Mt. near Jaśliska, 560 m, 22 Aug. 1974, J. Nowak (KRAM-L 33269). Gf 24 - Pasmo Bukowicy Mts., Graniczna stream near Bukowsko, 560 m, 24 Oct. 1974, J. Nowak (KRAM-L 33465); Pasmo Bukowicy Mts., stream near Darów by Moszczaniec, 
460 m, 16 Sept. 1974, J. Nowak (KRAM-L 33413); Darów near Wisłok Dolny, stream on S slope of Skibce Mt., 520 m, 16 Sept. 1974, J. Nowak (KRAM-L 33428). Gf 25 - Pasmo Bukowicy Mts., Nagórzany near Bukowsko, Sanoczek stream, 425 m, 24 Oct. 1974, J. Nowak (KRAM-L 33463). Gf 31 - Huta Polańska near Polana, 420 m, 10 Oct. 1979, J. Nowak (KRAM-L 32642). Gf 33 - Wola Wyżna near Jaśliska, Jasiołka stream, 510 m, 14 Sept. 1974, J. Nowak (KRAM-L 32970, 33414). Gf 34 - near Wisłok Górny, small stream between Wisłok river and Jamiska stream 650 m, 22 June 1974, J. Nowak (KRAM-L 33234, 33325). Gf 35 - Pasmo Bukowicy Mts., Izwór stream near Wisłok Wielki, 550 m, 23 June 1974, J. Nowak (KRAM-L 33408). Gf 44 - Wisłok Wielki Górny, Jamiska stream, W slope of Pasika Mt., 675 m, 22 June 1974, J. Nowak (KRAM-L 33231); Dołżyca near Komańcza, stream between Średni Garb Mt. and Danawa Mt., 650 m, 20 June 1974, J. Nowak (KRAM-L 33237); Wisłok Górny, Wisłok river, near Kanasiówka Mt., 625 m, 22 June 1974, J. Nowak (KRAM-L 33309).

Verrucaria procopii Servít - Gf 14 - Sieniawa by Wisłok river, 340 m, 4 Sept. 1977, J. Nowak (KRAM-L 23010). Gf 34 - near Wisłok Wielki, between Kiczera and Tokarnia peaks, 570 m, 19 June 1974, J. Nowak (KRAM-L 33242).

Verrucaria rupestris Schrad. - Ge 17 - Kąclowa, 16 July 1926, J. Motyka (LBL). Gf 20 - Kotań near Krempna, 390 m, 17 Sept. 1979, J. Nowak (KRAM-L 32886). Nieznajowa, 460 m, 9 Oct. 1979, J. Nowak (KRAM-L 32252). Gf 21 - Ostryszne near Krempna, 340 m, 19 Sept. 1979, J. Nowak (KRAM-L 33477).

Verrucaria sublobulata Eitner ex Servít - Gf 20 - Żydowskie near Krempna, strem on S slope of Cyrla Mt., 540 m, 21 Sept. 1979, J. Nowak (KRAM-L 32619). Gf 23 - Królik Włoski near Królik Polski, 510 m, 11 Oct. 1974, J. Nowak (KRAM-L 33284). Gf 44 - Wisłok Wielki Górny, stream Jamiska on W slope of Pasika Mt., 675 m, 22 June 1974, J. Nowak (KRAM-L 33231).

Verrucaria submersella Servít - Ge 17 - Kąclowa, 21 July 1926, J. Motyka (LBL). Verrucaria umbrinula Nyl. - Ge 17 - Kąclowa, 21 July 1926, J. Motyka (LBL). Verrucaria viridula (Schrad.) Ach. - Ge 17 - Kąclowa, 21 July 1926, J. Motyka (LBL).

Porosty rodzaju Verrucaria z obszaru Beskidu Niskiego

Streszczenie

W zbiorach zielnikowych KRAM i LBL z Beskidu Niskiego stwierdzono 26 gatunków Verrucaria s.l. (por. Appendix). V. aethiobola, V. andesiatica, V. aquatilis, V. dolosa, V. elaeina, V. elaeomelaena, V. funckii, V. hydrela, V. latebrosa, V. margacea, V.minuta, V. muralis, V. murina, $V$. nigrescens, V. ochrostoma, V. obfuscans, V. polysticta, V. praetermissa, V. rupestris, V. sublobulata, V. submersella, V. umbrinula, V. viridula oraz Hydropunctaria rheitrophila i Parabagliettoa cyanea (Gueiden et al. 2009) i Placopyrenium fuscellum (Navarro-Rosinés et al. 2007) wyróżniono w kolekcji zielnikowej J. Nowaka (KRAM) ze wschodniej części Beskidu Niskiego i kolekcji J. Motyki (LBL) z terenu Kąclowej koło Grybowa. Po raz pierwszy dla Polski podano gatunek Verrucaria elaeina. Pozostałe gatunki, z wyjątkiem V. nigrescens, V. polysticta i P. fuscellum (Olech 1974; Krzewicka 2009) zostały podane jako nowe dla tego terenu. 